\title{
Daphnetin inhibits inflammation in the NZB/W F1 systemic lupus erythematosus murine model via inhibition of NF- $\mathrm{KB}$ activity
}

\author{
MIN LI, XIAOWEI SHI, FANGRU CHEN and FEI HAO \\ Department of Dermatology, Southwest Hospital, Third Military Medical University, Chongqing 400038, P.R. China
}

Received August 22, 2015; Accepted October 21, 2016

DOI: 10.3892/etm.2016.3971

\begin{abstract}
Daphnetin is a compound extracted from Chinese medicinal herbs, which exerts analgesic and anti-inflammatory effects. The present study aimed to investigate the potential therapeutic effect of daphnetin on inflammation in the NZB/W F1 systemic lupus erythematosus (SLE) murine model. Female NZB/WF1 mice (age, 16-18 weeks) were intraperitoneally injected with daphnetin once a day for 12 weeks. It was revealed that daphnetin treatment significantly increased animal survival rates, reduced renal damage and blood urea nitrogen levels, and suppressed serum autoantibody production in the SLE-prone NZB/W F1 mice. In addition, daphnetin treatment significantly decreased the serum levels of tumor necrosis factor- $\alpha$ and interleukin-6, inhibited nuclear factor (NF)- $\kappa \mathrm{B}$ activity, suppressed the protein expression of nuclear factor of activated T-cells and promoted A20 protein expression in SLE-prone NZB/W F1 mice. In conclusion, daphnetin inhibited inflammation in the NZB/W F1 murine SLE model via inhibition of NF- $\mathrm{BB}$ mediated by upregulation of A20.
\end{abstract}

\section{Introduction}

Systemic lupus erythematosus (SLE) is a chronic systemic autoimmune disease characterized by high serum levels of autoantibodies and a repeated course of relapse and remission with multisystem damage as a clinical manifestation (1). It was reported in 2006 that the overall 1-year prevalence rate for SLE was 92.9 per 100,000 in China and 24.7 per 100,000 persons in Nottingham, England (2). SLE is a disease predominantly occurring in women, particularly those of childbearing age (aged 20-40 years) (3). It is a connective tissue disease with variable severity, which is mediated by autoimmunity

Correspondence to: Professor Fei Hao, Department of Dermatology, Southwest Hospital, Third Military Medical University, 29 Gaotanyan Centre Street, Chongqing 400038, P.R. China E-mail: haohaofeicq@163.com

Key words: daphnetin, systemic lupus erythematosus, inflammation, A20, nuclear factor- $\kappa \mathrm{B}$ with the characteristic symptom being immune-associated inflammation with multiple system and organ involvement (4).

The development of SLE may be associated with several pathogenic factors, including genetic and environmental factors as well as sex hormones (5). SLE is now thought to be caused by immune complexes formed in organs by autoantibodies secreted by activated $\mathrm{T}$ and $\mathrm{B}$ lymphocytes, particularly B lymphocytes after immunological abnormalities occur in the body through the interaction of the genetic background with environmental factors, sex hormones or infection (6-8).

The role of nuclear factor (NF)- $\kappa \mathrm{B}$ and its associated apoptotic cell signal transduction pathways are an international research hotspot. Studies have revealed that $\mathrm{NF}-\kappa \mathrm{B}$ inhibits apoptosis via interacting with various signaling molecules, including inhibitors of apoptosis, B-cell lymphoma 2, tumor necrosis factor receptor associated factors, c-Jun N-terminal kinase, Cellular FLICE-like inhibitory protein (c-FLIP), A20, growth arrest, DNA damage inducible beta and manganese superoxide dismutase, though mechanisms that have not been fully elucidated. Induction of apoptosis by inhibiting the activation of $\mathrm{NF}-\kappa \mathrm{B}$ signal transduction pathways may represent a novel treatment of autoimmune disease, inflammation and tumor (9). Moreover, with the elucidation of the critical role of NF- $\kappa \mathrm{B}$ subunits in apoptosis, novel strategies for treatment have been provided (10).

Daphnetin (Fig. 1), a coumarin derivative extracted from Chinese medicinal herbs (Daphne genus; Daphne Korean Nakai), has various pharmacological activities such as anti-inflammatory, anticoagulant, antibacterial, antifungal, antiviral, anticancer, antihypertensive, antitubercular, anticonvulsant, antiadipogenic, antihyperglycemic, antioxidant and neuroprotective properties (11). In China, daphnetin is used in the clinic as an oral Traditional Chinese Medicine (TCM), mainly for treating of arthritis, including rheumatoid arthritis, thromboangiitis obliterans and stenocardia (12). In the present study, the potential therapeutic effects of daphnetin on inflammation were investigated in the NZB/W F1 systemic lupus erythematosus (SLE) murine model.

\section{Materials and methods}

Animals and grouping. Female NZB/WF1 $(\mathrm{n}=40)$ and BALB/c mice $(n=10)$ (age, 16-18-weeks; weight, 35-45 g) 
were obtained from the Experimental Animal Centre of the Third Military Medical University (Chongqing, China) and housed in a specific pathogen-free barrier facility in our laboratory at $25 \pm 2^{\circ} \mathrm{C}$ and $40-70 \%$ relative humidity with a 12-h day/night lighting cycle and free access to standard rodent chow and filtered water. The animals were randomly assigned into three groups: NZB/W F1 mice with untreated SLE (SLE group; $n=20$ ), NZB/W F1 mice with SLE treated with daphnetin (daphnetin group; $\mathrm{n}=20$ ), and $\mathrm{BALB} / \mathrm{c}$ mice (control group; $\mathrm{n}=10$ ). Mice in the daphnetin group were intraperitoneally injected $5 \mathrm{mg} / \mathrm{kg}$ daphnetin in a total volume of $100 \mu 1$ once a day for 12 weeks. Control group and SLE group mice were intraperitoneally injected $100 \mu 1$ saline once a day for 12 weeks. Blood samples were obtained by retro-orbital bleeding from mice under anesthesia. At the end of the treatment, all mice were sacrificed while under isoflurane (Hebei Yipin Pharmaceutical Co. Ltd, Shijiazhuang, China; 1.5-3\% for $1 \mathrm{~min}$ ) inhaled anesthesia. The experimental protocol was proved by the Ethics Committee of the Third Military Medical University (Chongqing, China).

Assessment of renal function. The blood samples collected from mice were clotted at room temperature. The samples were centrifuged at $11,200 \times \mathrm{g}$ for $5 \mathrm{~min}$ and the supernatants were collected to analyze blood urea nitrogen (BUN) levels using the Urea Nitrogen Diacetylmonoxime Test kit (Tiangen, Beijing, China).

Serum levels of anti-nuclear-ribonuclear-protein (nRNP) immumoglobulin $G(\operatorname{Ig} G)$ and anti-double-stranded (ds) $D N A \operatorname{Ig} G$. The blood samples collected from mice were centrifuged at 11,200 $\mathrm{x} g$ for $5 \mathrm{~min}$ and the supernatants were collected as serum. Serum levels of anti-nRNP IgG and anti-dsDNA IgG were measured using a Mouse Anti-nRNP IgG ELISA kit (Alpha Diagnostic, San Antonio, TX, USA) and Mouse Anti-dsDNA IgG ELISA kit (Alpha Diagnostic). In brief, serum samples (50 $\mu \mathrm{l}$ each) were added in triplicate into 96-well plates which had been coated with specific antibodies and incubated at room temperature for $2 \mathrm{~h}$. Following three washes with phosphate-buffered saline containing Tween 20, the bound antigens were detected by incubation with biotinylated antibodies at room temperature for $30 \mathrm{~min}$. Subsequently, the bound biotinylated antibodies were detected with horseradish peroxidase (HRP)-conjugated streptavidin and chromogenic reagent, followed by reading the absorbance at $450 \mathrm{~nm}$ in an ELISA reader (ELx800; BioTek Instruments, Inc., Winooski, VT, USA).

Serum levels of tumor necrosis factor (TNF)- $\alpha$ and interleukin (IL)-6. The serum was kept at $-20^{\circ} \mathrm{C}$ until tested. Serum levels of TNF- $\alpha$ and IL- 6 were measured using a Mouse Anti-TNF- $\alpha$ IgG ELISA kit (Invitrogen/BioSource International, Camarillo, CA, USA) and Mouse Anti-IL-6 IgG ELISA kit (Invitrogen/BioSource International. The reaction was monitored spectrophotometrically by measuring the absorbance at $450 \mathrm{~nm}$ using the ELx800 ELISA reader.

Assessment of phosphorylated (p)NFKB-p65, nuclear factor of activated T-cells (NFAT) and A20 protein levels using western blot analysis. Mouse peripheral blood lymphocytes were isolated from peripheral blood by density gradient centrifugation ( $800 \times \mathrm{x}$, 20-30 min) using mouse lymphocyte separation medium (Dakewe, Inc., Shenzhen, China) and suspended in RPMI-1640 medium (Gibco; Thermo Fisher Scientific, Inc., Waltham, MA, USA) containing 10\% heat-inactivated fetal calf serum (Gibco; Thermo Fisher Scientific, Inc.), $100 \mathrm{U} / \mathrm{ml}$ penicillin and $100 \mu \mathrm{g} / \mathrm{ml}$ streptomycin. Mouse peripheral blood lymphocytes were lysed in Beyotime Lysis Buffer (Beyotime Institute of Biotechnology, Haimen, China) and homogeneously mixed with lysis buffer, followed by centrifugation at $4^{\circ} \mathrm{C}, 15,000 \mathrm{x} g$ for $10 \mathrm{~min}$. Cell lysate supernatant was quantified using BCA Protein Assay Reagent kit (Beyotime Institute of Biotechnology, Beijing, China). The sample proteins were separated using 10-12\% SDS-PAGE (50 $\mu \mathrm{g} /$ lane) and then transferred onto a polyvinylidene difluoride (PVDF) membrane (EMD Millipore, Billerica, MA, USA). After blocking with 5\% skimmed milk powder in Tris-buffered saline containing Tween 20 (TBST), the membranes were incubated overnight at $4^{\circ} \mathrm{C}$ with the following primary antibodies: Anti-A20 (ab74037; 1:500; Abcam, Cambridge, UK), anti- $\beta$-actin (ab8227; 1:1,000; Abcam), anti-NFAT (ab25916; 1:1,000; Abcam), anti-GADPH (sc25778; 1;1,000; Santa Cruz Biotechnology, Inc., Dallas, TX, USA), anti-NF-кB-p65 (sc7151; 1:600; Santa Cruz Biotechnology, Inc.) and anti-pNF-кB-p65 (sc101749; 1:600; Santa Cruz Biotechnology, Inc.). After rinsing briefly with TBST buffer (5 $\min \times 3$ ), the bound antibodies were detected with HRP-conjugated goat anti-rabbit IgG secondary antibodies (sc2301; 1:1,000, Santa Cruz Biotechnology, Inc.) for $1 \mathrm{~h}$ and reacting bands were revealed by a ECL reagent (P0019; Beyotime Institute of Biotechnology) and photographed using a chemiluminescence imaging system (ChemiDoc XRS+; Bio-Rad Laboratories, Inc., Hercules, CA, USA). The amount of protein in each band was quantified using Quantity One 4.6 software (Bio-Rad Laboratories, Inc.).

Statistical analysis. All values are expressed as the mean \pm standard deviation. Differences between groups were analyzed using the Mann-Whitney U test or one-way analysis of variance if the data were normally distributed. $\mathrm{P}<0.05$ was considered to indicate a statistically significant difference.

\section{Results}

Daphnetin increases the survival rate of mice with SLE. The survival rate in each group was determined over the course of the experiment. The survival rate of the NZB/WF1 mice in the SLE group was lower than that of the BALB/c mice in the control group (Fig. 2). Of note, daphnetin treatment clearly increased the survival of NZB/WF1 mice with SLE (Fig. 2).

Daphnetin reduces SLE-associated renal damage in mice. To determine the therapeutic effects of daphnetin on renal injury associated with SLE, BUN levels were analyzed in each study group. In the in NZB/WF1 mice in the SLE group, BUN levels were significantly increased compared with the $\mathrm{BALB} / \mathrm{c}$ mice in the control group, indicating that the mice in the SLE group had suffered renal damage (Fig. 3). Of note, 
daphnetin treatment significantly reduced BUN levels and therefore renal damage in NZB/WF1 mice.

Daphnetin treatment mitigates humoral responses in mice with SLE by decreasing anti-nRNP IgG and anti-dsDNA $I g G$. To evaluate the impact of daphnetin on the pathogenesis of SLE, the serum levels of anti-nRNP IgG and anti-dsDNA $\mathrm{IgG}$ in mice were assessed using ELISA. The results revealed that the serum levels of anti-nRNP IgG in mice in the SLE group were significantly higher than those in the control group. As expected, the serum levels of anti-nRNP IgG in the daphnetin group were significantly lower than those in the SLE group (Fig. 4A). A similar pattern of serum anti-dsDNA IgG levels was observed in the three groups (Fig. 4B). Thus, it is indicated that daphnetin treatment mitigated humoral responses in NZB/WF1 SLE-prone mice.

Daphnetin reduces the serum levels of TNF- $\alpha$ and IL- 6 in mice with SLE. In order to investigate the protective effects of daphnetin on serum cytokine levels, TNF- $\alpha$ and IL- 6 were measured using mouse cytokine kits. Of note, NZB/WF1 mice in the SLE group had significantly higher serum levels of TNF- $\alpha$ and IL- 6 than the BALB/c mice in the control group (Fig. 5). Furthermore, daphnetin significantly reduced the serum levels of TNF- $\alpha$ and IL-6 in NZB/WF1 mice as compared with those in the SLE group (Fig. 5).

Daphnetin reduces $N F-\kappa B$ activity in mice. The present study further investigated the mechanism by which daphnetin exerts its anti-inflammatory effects in mice with SLE. The protein levels of NF- $\mathrm{B}-\mathrm{p} 65$ and $\mathrm{pNF}-\kappa \mathrm{B}-\mathrm{p} 65$ in the peripheral blood lymphocytes of mice were analyzed by western blot to assess $\mathrm{NF}-\kappa \mathrm{B}$ activity. Indeed, the protein levels of pNF-кB-p65 in the SLE model group were substantially increased compared with those in the control group (Fig. 6). Furthermore, daphnetin treatment significantly reduced the levels of $\mathrm{pNF}-\kappa \mathrm{B}-\mathrm{p} 65$ in NZB/WF1 mice.

Daphnetin reduces NFAT protein expression in mice with $S L E$. In order to further investigate the mechanism of action of daphnetin, its effect on NFAT proteins, which are expressed in most cells of the immune system and have a pivotal role in the transcription of genes encoding cytokines and other proteins critical for the immune response, was assessed. The results showed a marked increase of NFAT protein expression in the peripheral blood lymphocytes of mice in the SLE model group compared with that in the control group (Fig. 7). Furthermore, daphnetin significantly reduced the SLE-induced NFAT protein expression in NZB/WF1 mice.

A20 protein expression in mice. A20, also known as tumor necrosis factor $\alpha$-induced protein 3 (TNFAIP3), is an anti-inflammatory factor which is induced by TNF- $\alpha$ and abrogated by NF- $\kappa \mathrm{B}$ activation (13). To further understand the protective effects of daphnetin on SLE, the protein expression of A20 in the peripheral blood lymphocytes of NZB/WF1 mice was assessed by western blot analysis. As shown in Fig. 8, A20 protein expression was significantly elevated in the SLE group compared with that in the control group. In addition, daphnetin treatment significantly promoted A20

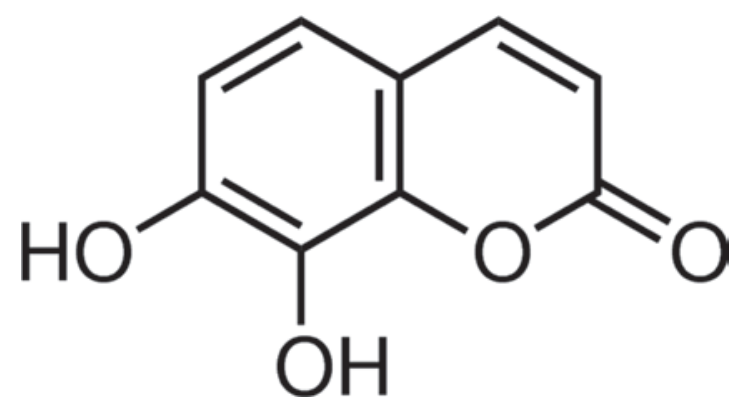

Figure 1. Chemical structure of daphnetin.

protein expression in NZB/WF1 mice compared with that in the SLE group (Fig. 8).

\section{Discussion}

SLE is a systemic autoimmune disease with various pathogenic factors and multiple organ involvement as well as diverse clinical manifestations and a relapsing and remitting course (1). SLE occurs globally, while a diversity in its prevalence, morbidity and disease severity has been observed among different regions and ethnicities (2). SLE occurs at any age, but mainly affects in women of childbearing age, with the incidence rate in women being 6-fold higher than that in men (14). Although the pathogenesis of SLE remains to be fully elucidated, it is commonly thought that SLE lesions in tissues and organs are caused by the deposited immune complexes formed by the autoantibodies secreted by abnormally activated $\mathrm{T}$ and $\mathrm{B}$ lymphocytes after the autoimmune tolerance is broken due to the occurrence of immunological abnormalities in patients with genetic susceptibility under the stimulation by other risk factors (4). Daphnetin, a coumarin derivative, has therapeutic efficacy against autoimmune arthritis through modulating the balance of T-regulatory cells and T helper 17 cells (15). Shu et al (16) reported that daphnetin exerts its effects against autoimmune arthritis via demethylation of pro-apoptotic genes in synovial cells. The present study found that daphnetin treatment significantly increased the survival rate, reduced renal damage and BUN levels, and suppressed serum autoantibody production in SLE-prone NZB/W F1 mice.

$\mathrm{NF}-\kappa \mathrm{B}$ signaling pathways are activated by numerous stimuli, including cytokines, B- and T-cell receptor homologous antigens and oxidative stress, triggering a series of signaling cascades that activate $\mathrm{I} \kappa \mathrm{B}$ kinase, which then

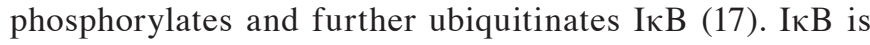
degraded through proteasome-associated processes, resulting in the release of $\mathrm{NF}-\kappa \mathrm{B}$ into the cell nucleus to regulate the transcription of target genes after binding with gene promoters (18). The products of the target genes are usually involved in the regulation of the inflammatory and immune response, as well as growth, proliferation, differentiation and apoptosis of immunocytes (18). It has been indicated that aberrations in $\mathrm{NF}-\kappa \mathrm{B}$ signaling lead to abnormal inflammatory and immune responses, showing a close association between abnormal NF- $\mathrm{KB}$ activation and the occurrence and development of chronic inflammation, tumors and SLE (19). In line with this, the present study found that daphnetin 


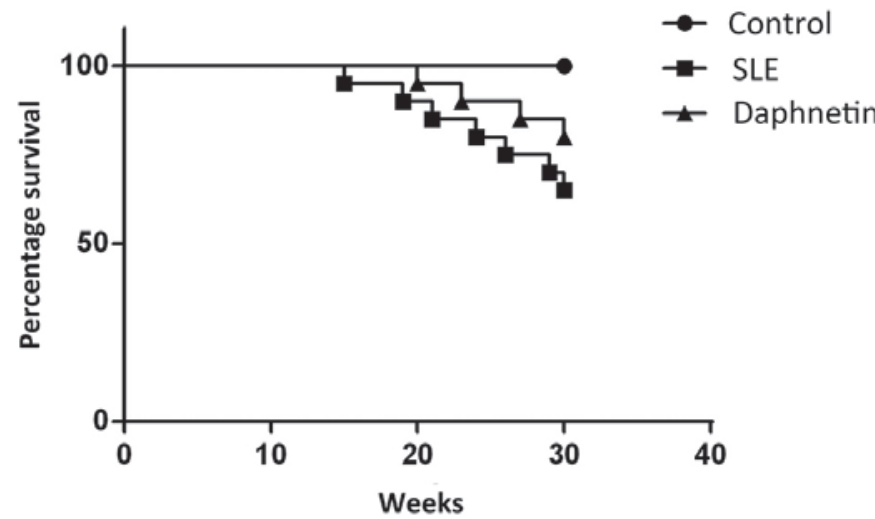

Figure 2. Animal survival analysis in each study group. Comparison of the Kaplan-Meier curves for each study group (SLE group, $n=20$; daphnetin group, $\mathrm{n}=20$; control group; $\mathrm{n}=10$ ). SLE, systemic lupus erythematosus.

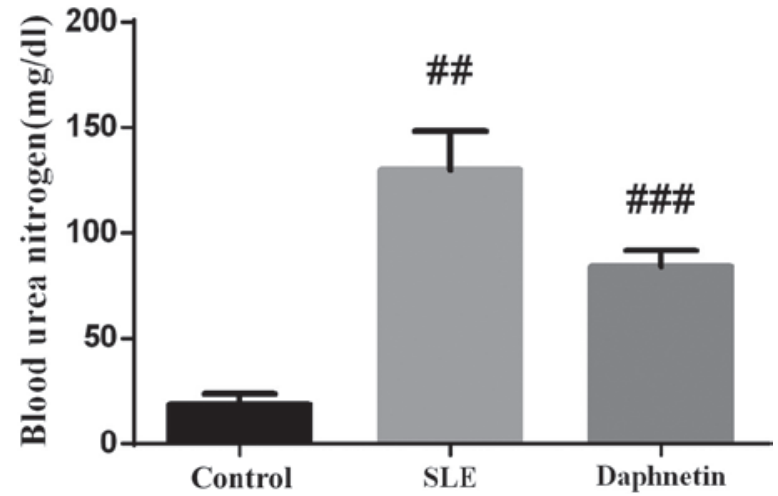

Figure 3. BUN levels in each study group determined by ELISA. Values are expressed as the mean \pm standard deviation (SLE group, $n=20$; daphnetin group, $\mathrm{n}=20$; control group; $\mathrm{n}=10)$. ${ }^{\# \#} \mathrm{P}<0.01$ vs. control group; ${ }^{\# \#} \mathrm{P}<0.01$ vs. SLE model group. BUN, blood urea nitrogen; SLE, systemic lupus erythematosus.
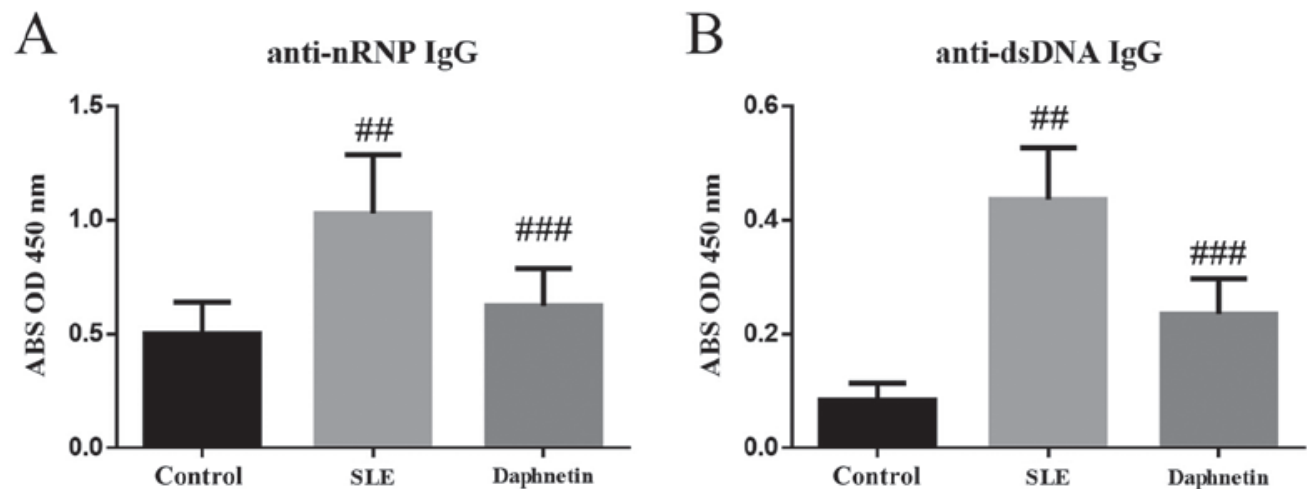

Figure 4. Serum levels of (A) anti-nRNP IgG and (B) anti-dsDNA IgG in mice in the three groups determined by ELISA. Values are expressed as the mean \pm standard deviation (SLE group, $n=20$; daphnetin group, $n=20$; control group; $n=10$ ). ${ }^{\# \#} \mathrm{P}<0.01$ vs. control group; ${ }^{\# \# /} \mathrm{P}<0.01$ vs. SLE model group. IgG, immunoglobulin G; dsDNA, double-stranded DNA; nRNP, nuclear-ribonuclear-protein; SLE, systemic lupus erythematosus; ABS OD, absorbance optical density.
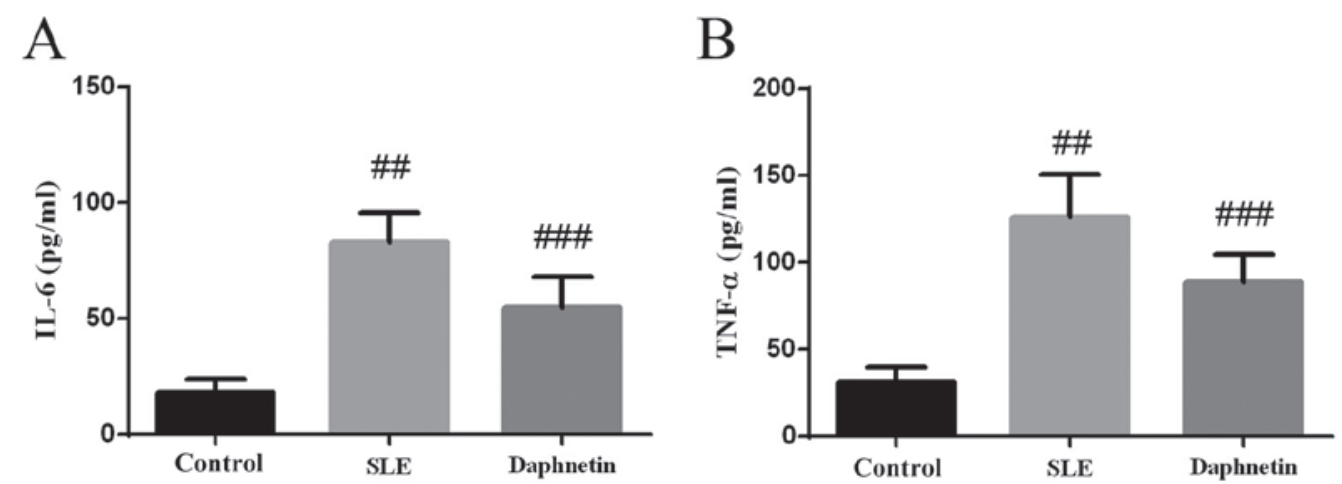

Figure 5. Serum levels of (A) TNF- $\alpha$ and (B) IL-6 the serum of mice in the three groups determined by ELISA. Values are expressed as the mean \pm standard deviation (SLE group, $n=20$; daphnetin group, $n=20$; control group; $n=10$ ). ${ }^{\# \#} \mathrm{P}<0.01$ vs. control group; ${ }^{\# \#} \mathrm{P}<0.01$ vs. SLE model group. SLE, systemic lupus erythematosus; TNF, tumor necrosis factor; IL, interleukin.

treatment significantly decreased the serum levels of TNF- $\alpha$ and IL-6, inhibited NF- $\mathrm{B}$ activity, suppressed NFAT protein expression and promoted A20 protein expression in SLE-prone NZB/W F1 mice. Song et al (20) also revealed that daphnetin has marked immunosuppressive activity and is mediated via inhibition of NF- $\mathrm{BB}$ and NFAT signaling pathways in mouse T cells. Yu et al (21) demonstrated that daphnetin exerted anti-inflammatory and protective effects against endotoxin-induced lung injury by decreasing NF- $\mathrm{KB}$ activity through modulation of A20.

Protein A20, also known as TNFAIP3, is a zinc finger protein with high bioactivity, and mutations in A20 are associated with autoimmune/inflammatory diseases such as coronary artery disease in type 2 diabetes, rheumatoid 

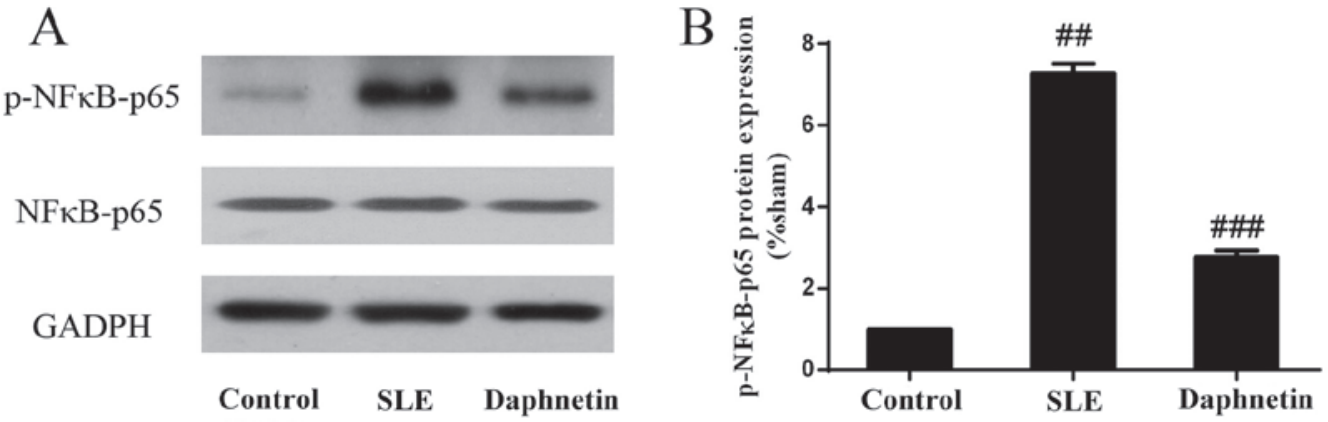

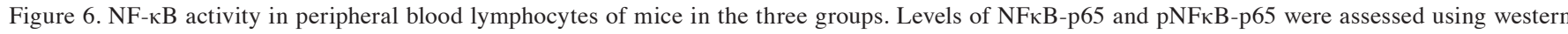
blot analysis. (A) A representative gel is shown. (B) Quantification by densitometric analysis showing NF- $\mathrm{\kappa B}$ activity in mice. Values are expressed as the mean \pm standard deviation (SLE group, $n=20$; daphnetin group, $n=20$; control group; $n=10$ ). ${ }^{\# \# ~} \mathrm{P}<0.01$ vs. control group; ${ }^{\# \# "} \mathrm{P}<0.01$ vs. SLE model group. $\mathrm{p}-\mathrm{NF}$, phosphorylated nuclear factor; SLE, systemic lupus erythematosus.
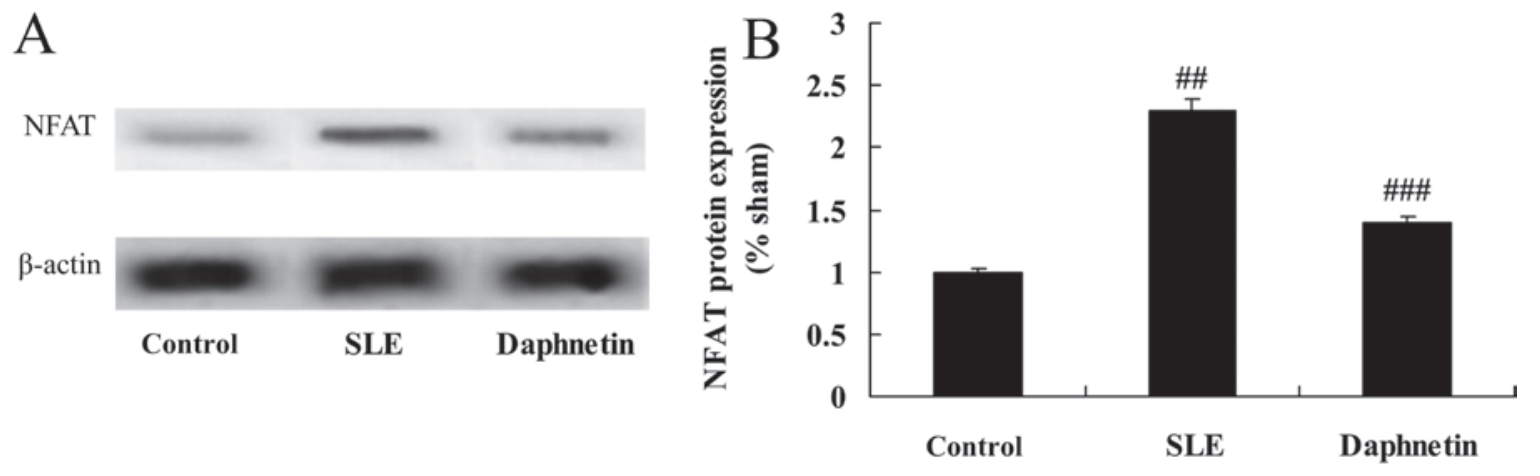

Figure 7. NFAT protein expression in peripheral blood lymphocytes of mice in the three groups determined by western blot analysis. (A) A representative gel is shown. (B) Protein expression was quantified by densitometric analysis. Values are expressed as the mean \pm standard deviation (SLE group, $n=20$; daphnetin group, $\mathrm{n}=20$; control group; $\mathrm{n}=10) .{ }^{\# \#} \mathrm{P}<0.01$ vs. control group; ${ }^{\# \# \#} \mathrm{P}<0.01$ vs. SLE model group. SLE, systemic lupus erythematosus; NFAT, nuclear factor of activated T-cells.
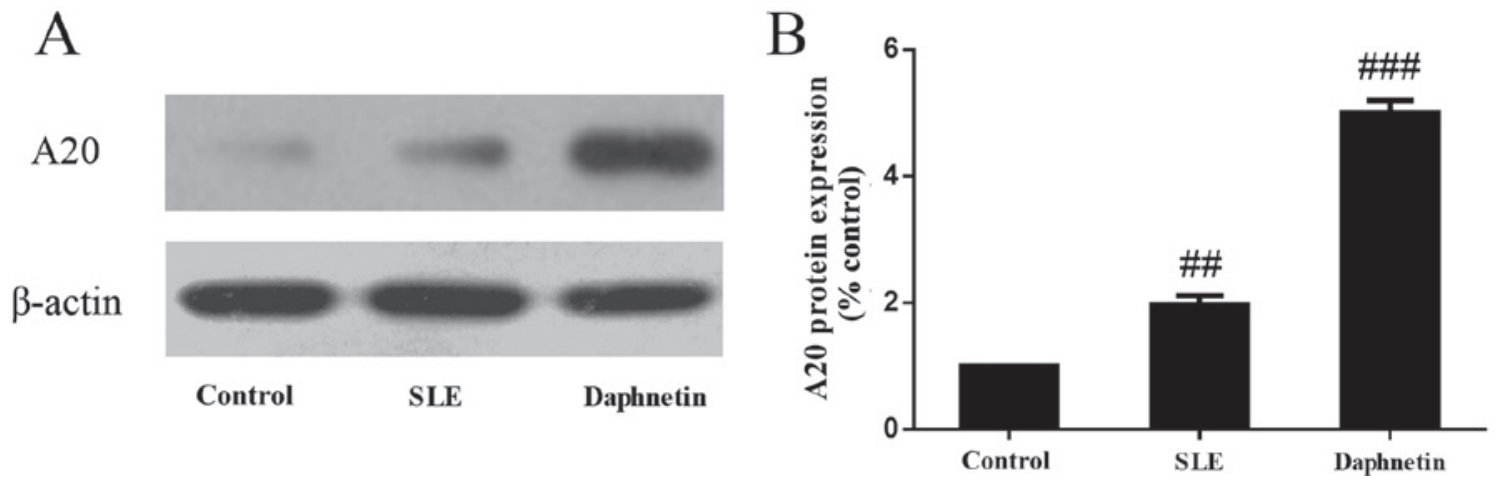

Figure 8. A20 protein expression in peripheral blood lymphocytes of mice in the three groups determined by western blot analysis. (A) A representative gel is shown. (B) Protein expression was quantified by densitometric analysis. Values are expressed as the mean \pm standard deviation (SLE group, $\mathrm{n}=20$; daphnetin group, $\mathrm{n}=20$; control group; $\mathrm{n}=10$ ). ${ }^{\# \#} \mathrm{P}<0.01$ vs. control group; ${ }^{\# \#} \mathrm{P}<0.01$ vs. SLE model group. SLE, systemic lupus erythematosus.

arthritis, SLE, type 1 diabetes, chronic rhinosinusitis, psoriasis, celiac disease and inflammatory bowel disease, rheumatic heart disease, systemic sclerosis and Behcet disease (22). A20 has a critical function in abrogating the activation of $\mathrm{NF}-\kappa \mathrm{B}$ (23). Furthermore, NF- $\kappa \mathrm{B}$ modulates TNF- $\alpha$-induced apoptosis by regulating the expression of A20 (17), which in turn inhibits the activation of NF- $\mathrm{B}$ in several inflammatory diseases, including bronchial asthma, rheumatoid arthritis, atherosclerosis, myocarditis and hepatitis (24). Notably, the results of the present study showed that daphnetin exerted its protective effects by significantly promoting SLE-induced A20 protein expression in SLE-prone NZB/WF1 mice.

In conclusion, the present study demonstrated the protective effect of daphnetin against inflammation in the NZB/W F1 murine SLE model. The results suggested that the therapeutic effects of daphnetin may be associated with the downregulation of $\mathrm{NF}-\kappa \mathrm{B}$ signaling mediated by upregulation of A20. The present study therefore indicated that daphnetin is a potential treatment option for patients with SLE. 


\section{Acknowledgements}

This study was supported by the National Natural Science Foundation of China (grant no. 81271753).

\section{References}

1. Choi J, Kim ST and Craft J: The pathogenesis of systemic lupus erythematosus-an update. Curr Opin Immunol 24: 651-657, 2012.

2. Lau CS, Yin G and Mok MY: Ethnic and geographical differences in systemic lupus erythematosus: an overview. Lupus 15: 715-719, 2006.

3. Sadowska A, Hebanowski M, Trzeciak B, Kliszcz J, Nowicka-Sauer K and Waldman M: Retrospective evaluation of pregnancy in women suffering from systemic lupus erythematosus (SLE). Ginekol Pol 76: 955-959, 2005 (In Polish).

4. Liu Z and Davidson A: Taming lupus-a new understanding of pathogenesis is leading to clinical advances. Nat Med 18: 871-882, 2012.

5. Cohen-Solal JF, Jeganathan V, Grimaldi CM, Peeva E and Diamond B: Sex hormones and SLE: Influencing the fate of autoreactive B cells. Curr Top Microbiol Immunol 305: 67-88, 2006.

6. Molad Y, Pokroy-Shapira E, Kaptzan T, Monselise A, Shalita-Chesner M and Monselise Y: Serum soluble triggering receptor on myeloid cells-1 (sTREM-1) is elevated in systemic lupus erythematosus but does not distinguish between lupus alone and concurrent infection. Inflammation 36: 1519-1524, 2013.

7. Hedrich CM, Crispin JC and Tsokos GC: Epigenetic regulation of cytokine expression in systemic lupus erythematosus with special focus on T cells. Autoimmunity 47: 234-241, 2014.

8. Marquart HV, Svendsen A, Rasmussen JM, Nielsen CH, Junker P, Svehag SE and Leslie RG: Complement receptor expression and activation of the complement cascade on $\mathrm{B}$ lymphocytes from patients with systemic lupus erythematosus (SLE). Clin Exp Immunol 101: 60-65, 1995.

9. Park MH and Hong JT: Roles of NF- $\mathrm{NB}$ in cancer and inflammatory diseases and their therapeutic approaches. Cells 5: E15, 2016.

10. Hoesel B and Schmid JA: The complexity of $\mathrm{NF}-\kappa \mathrm{B}$ signaling in inflammation and cancer. Mol Cancer 12: 86, 2013.

11. Venugopala KN, Rashmi V and Odhav B: Review on natural coumarin lead compounds for their pharmacological activity. Biomed Res Int 2013: 963248, 2013.
12. Fa ZM: Advance in pharmacological and clinical research of daphnetin. Modern Diagnosis \& Treatment: 138-141, 1992.

13. Opipari AW Jr, Boguski MS and Dixit VM: The A20 cDNA induced by tumor necrosis factor alpha encodes a novel type of zinc finger protein. J Biol Chem 265: 14705-14708, 1990.

14. Ruo VHMC: The epidemiology of systemic lupus erythematosus. Dubois' Lupus Erythematosus. Philadelphia, Lippincott, Williams \& Wilkins, pp65-83, 2002.

15. Tu L, Li S, Fu Y, Yao R, Zhang Z, Yang S, Zeng X and Kuang N: The therapeutic effects of daphnetin in collagen-induced arthritis involve its regulation of Th17 cells. Int Immunopharmacol 13: 417-423, 2012.

16. Shu K, Kuang N, Zhang Z, Hu Z, Zhang Y, Fu Y and Min W: Therapeutic effect of daphnetin on the autoimmune arthritis through demethylation of proapoptotic genes in synovial cells. J Transl Med 12: 287, 2014.

17. Pujari R, Hunte R, Khan WN and Shembade N: A20-mediated negative regulation of canonical $N F-\kappa B$ signaling pathway. Immunol Res 57: 166-171, 2013.

18. Hayden MS and Ghosh S: NF- $\kappa$ B, the first quarter-century: Remarkable progress and outstanding questions. Genes Dev 26 203-234, 2012

19. Jolly CA, Muthukumar A, Reddy Avula CP and Fernandes G: Maintenance of NF-kappaB activation in T-lymphocytes and a naive T-cell population in autoimmune-prone (NZB/NZW)F(1) mice by feeding a food-restricted diet enriched with n-3 fatty acids. Cell Immunol 213: 122-133, 2001.

20. Song B, Wang Z, Liu Y, Xu S, Huang G, Xiong Y, Zhang S, $\mathrm{Xu} \mathrm{L}$, Deng $\mathrm{X}$ and Guan S: Immunosuppressive activity of daphnetin, one of coumarin derivatives, is mediated through suppression of $\mathrm{NF}-\kappa \mathrm{B}$ and NFAT signaling pathways in mouse T cells. PLoS One 9: e96502, 2014.

21. Yu WW, Lu Z, Zhang H, Kang YH, Mao Y, Wang HH, Ge WH and Shi LY: Anti-inflammatory and protective properties of daphnetin in endotoxin-induced lung injury. J Agric Food Chem 62: 12315-12325, 2014.

22. Mele A, Cervantes JR, Chien V, Friedman D and Ferran C: Single nucleotide polymorphisms at the TNFAIP3/A20 locus and susceptibility/resistance to inflammatory and autoimmune diseases. Adv Exp Med Biol 809: 163-183, 2014

23. Verstrepen L, Carpentier I and Beyaert R: The biology of A20-binding inhibitors of NF-kappaB activation (ABINs). Adv Exp Med Biol 809: 13-31, 2014.

24. Catrysse L, Vereecke L, Beyaert R and van Loo G: A20 in inflammation and autoimmunity. Trends Immunol 35: 22-31, 2014. 